\title{
Routine Cystoscopy during Robotic Hysterectomy for Endometrial Cancer
}

\author{
Diana El-neemany ${ }^{1}$, Nicole Pursell ${ }^{1}$, Erin Curcio ${ }^{1}$, Alexandra Giglio ${ }^{1}$, Yu Chen ${ }^{1}$, Patricia Greenberg ${ }^{2}$, \\ Verda J Hicks ${ }^{3}$ and Karim Elsahwi ${ }^{3^{*}}$
}

${ }^{1}$ Department of Obstetrics and Gynecology, Jersey Shore University Medical Center, USA

${ }^{2}$ Office of Research Administration, Jersey Shore University Medical Center, USA

${ }^{3}$ Division of Gynecologic Oncology, Jersey Shore University Medical Center, USA

\begin{abstract}
Background: The use of routine cystoscopy after hysterectomy is controversial.

Objective: To evaluate the role of routine cystoscopy in the detection of urinary tract injuries in robotic total laparoscopic hysterectomies performed for endometrial cancer staging.

Study design: This was a retrospective chart review of patients undergoing robotic-assisted laparoscopic hysterectomy for endometrial cancer staging by a single gynecologic oncologist between January 2012 and December 2015. Routine cystoscopy was performed in all cases.

Results: A total of 157 cases met inclusion criteria and among those, five cases of urinary tract injury were identified (3.2\%). Among the patients with urinary tract injury, average age was 66 [range 47, 90] years old, the average BMI was 34 [range 25, 34], and all patients had Stage I of endometrial cancer. Three patient injuries were detected intraoperatively, and 2 injuries were diagnosed postoperatively. Two of the intraoperative injuries were caused by dissection of the bladder from the lower uterine segment and detected prior to performing the cystoscopy. Another intraoperative injury was detected immediately after performing a mini-laparotomy to extract a larger uterus. The 2 urinary tract injury cases detected postoperatively were both ureteral injuries. The visual detection rate of the urinary tract injuries was $60 \%(3 / 5)$, while the cystoscopy detection rate was $0 \%$. There were no significant differences detected in risk factors between the injury cases and the whole cohort.
\end{abstract}

Conclusion: In this study, the incidence of urinary tract injury in robotic hysterectomy performed for endometrial cancer staging was 3.2\%. Performing routine cystoscopy did not improve detection of urinary tract injuries in these cases.

\section{Keywords}

Cystoscopy, Endometrial cancer, Hysterectomy, Laparoscopy, Robot, Urinary tract injury

\section{Introduction}

Gynecologic surgery is associated with an increased risk of injury to the lower urinary tract. The overall estimated incidence of urinary tract injury when performing a hysterectomy ranges from 0.2 to 15 per 1000 cases [1]. More specifically, laparoscopic hysterectomy has higher rates of urinary tract injuries compared to other surgical routes, with an incidence of bladder injuries ranging between 8.9-12.1 per 1000 cases and an incidence of ureteral injuries between 7.3-13.9 per 1000 cases [2-4]. Though hysterectomy related urinary tract injuries are rare, the morbidity associated with delayed detection can be devastating, incurring emotional and financial cost to both the patient and the health care system [5]. Several studies have shown that routine cystoscopy can help increase detection rates of urinary tract injuries. Teeluckdharry and colleagues noted a 95\% increase in detection of ureteric or bladder injuries intraoperatively when using cystoscopy [6]. Another study by Chi and colleagues showed there were significantly fewer delayed urologic complications after a policy of universal cystoscopy

*Corresponding author: Karim Elsahwi, MD, FACOG, FACS, Division of Gynecologic Oncology, Hackensack Meridian Health, Jersey Shore University Medical Center, 1945 Corlies Avenue, Neptune, NJ, USA, Tel: 732-776-3790, Fax: 732-922-8264

Accepted: January 19, 2019

Published online: January 21, 2019

Citation: El-neemany D, Pursell N, Curcio E, et al. (2019) Routine Cystoscopy during Robotic Hysterectomy for Endometrial Cancer. Reports Gynecol Surg 2(1):12-17 
was instituted [7]. However, controversy still exists regarding the adoption of a universal cystoscopy policy as other studies have shown them to have no benefit in detecting urinary tract injury [8,9].

Robotic laparoscopic methods are growing in popularity in the field of gynecology [10]. Robotic-assisted surgery has also been shown to be a feasible method for complicated gynecological cases and is receiving more attention for use in different gynecologic malignancies due to its promising results [10-14]. Few studies have addressed the benefit of universal cystoscopy associated with robotic laparoscopic surgery in endometrial cancer staging $[9,11]$. The objective of this study was to evaluate the role of routine cystoscopy in the detection of urinary tract injuries in robotic total laparoscopic hysterectomies for endometrial cancer staging. The secondary objective was to evaluate potential risk factors that may increase the risk of urinary tract injury during those operations.

\section{Materials and Methods}

A retrospective chart review of patients who underwent robotic-assisted staging for endometrial cancer by one gynecologic oncologist in one healthcare system between January 2012 and December 2015 was performed. Patient inclusion into the study required that a total laparoscopic hysterectomy be performed for an endometrial malignancy. The da Vinci $\mathrm{Si}^{\circledR}$ robot was used as the platform for performing all the surgeries and routine cystoscopy was performed at the conclusion of each case. Even if an injury to the urinary tract was detected intraoperatively, a cystoscopy was still performed at the end of the procedure to confirm the integrity of the bladder repair and exclude any further ureter injury. Data collection included: demographic information such as age, Body Mass Index (BMI), past medical history related to cardiovascular or pulmonary systems, and any previous abdominal surgery. Pulmonary disease was defined as grade 2 or above restrictive or obstructive pulmonary disease based on the National Institutes of Health (NIH) Common Terminology Criteria for Adverse Events (CTACE 4.03). Cardiac morbidity was defined as grade 2 or above (CTACE 4.03) ischemic or valvular disease, heart failure or arrhythmia. Operative data abstracted included: Preoperative and postoperative diagnoses, length of surgery, intraoperative complications, total estimated blood loss, postoperative complications, readmissions and final pathology. Length of surgery was defined as the time from the initial incision to skin closure. This included docking of the robot, time spent on the robotic console, and cystoscopy. Cystoscopy was performed using a diagnostic 70-degree lens through a 17-French sheath in a systematic 360-degree exploration of the bladder including the dome, the trigone, and both ureteric orifices. Saline was used as a distending medium. Due to a national shortage of blue dye (indigo carmine), this was not consistently used across all cases. Cystoscopy was continued until efflux of urine from the ureteric orifices was confirmed bilaterally by the surgeon, both assistants and in most of the cases the scrub technician. Descriptive statistics, Fisher Exact tests, and Wilcoxon rank sum tests were used to draw conclusions in this study. All p-values reported are

two-sided and any p-value less than 0.05 was considered statistically significant. Institutional review board approval for this study was obtained through the Jersey Shore University Medical Center IRB in Neptune, New Jersey.

\section{Results}

A total of 220 robotic cases were reviewed; However, 63 cases where the patient did not have a total laparoscopic hysterectomy, or the post-operative findings revealed no endometrial malignancy were excluded, leaving 157 patients in the analytic data set. Average age at the time of surgery was 66 years old and the average BMI was $34 \mathrm{~kg} /$ $\mathrm{m}^{2}$. Most patients $(72.6 \%)$ had stage- 1 endometrial cancer. A full list of patient demographics is shown in Table 1 and

Table 1: Full sample patient characteristics $(n=157)$.

\begin{tabular}{|l|l|}
\hline Patient characteristic & Median [IQR] \\
\hline Age at surgery $($ years $)$ & $66.1[57.2,74.3]$ \\
\hline Body mass index $\left(\mathrm{kg} / \mathrm{m}^{2}\right)$ & $33[28,40]$ \\
\hline & Frequency (\%) \\
\hline History of CVD & $119(75.8)$ \\
\hline History of COPD & $42(26.8)$ \\
\hline Prior abdominal surgery & $76(48.4)$ \\
\hline EAC stage & $114(72.6)$ \\
\hline Stage 1 & $17(10.8)$ \\
\hline Stage 2 & $17(10.8)$ \\
\hline Stage 3 & $7(4.5)$ \\
\hline Stage 4 & $2(1.3)$ \\
\hline Unknown & \\
\hline Histopathology & $140(89.2)$ \\
\hline Type 1 & $17(10.8)$ \\
\hline Type 2 & \\
\hline Urinary tract injury type & $3(1.9)$ \\
\hline Bladder injury & $2(1.3)$ \\
\hline Ureter injury & \\
\hline
\end{tabular}

$\mathrm{IQR}=$ Interquartile range $; \mathrm{CVD}=$ Cardiovascular disease $; \mathrm{COPD}=$ Chronic obstructive pulmonary disease; $\mathrm{EAC}=$ Endometrial adenocarcinoma.

Table 2: Full sample operative data $(n=157)$.

\begin{tabular}{|l|l|}
\hline Primary procedure performed: & Frequency (\%) \\
\hline Total laparoscopic hysterectomy & $151(96.2)$ \\
\hline Radical laparoscopic hysterectomy & $6(3.8)$ \\
\hline Additional procedural stepst: & \\
\hline Bilateral salpingectomy & $2(1.3)$ \\
\hline Bilateral salpingo-oophorectomy & $155(98.7)$ \\
\hline Omentectomy & $29(18.5)$ \\
\hline Lysis of adhesions & $90(57.3)$ \\
\hline Pelvic lymph node dissection & $128(81.5)^{*}$ \\
\hline Para-aortic lymph node dissection & $94(59.9)^{*}$ \\
\hline Procedure measures: & Median [IQR] \\
\hline Uterine weight (g) & $140[96,188]^{*}$ \\
\hline Length of surgery (min) & $200[158,233]^{*}$ \\
\hline Estimated total blood loss (mL) & $100[100,150]^{*}$ \\
\hline \begin{tabular}{l} 
*Indicates missing data (less than 5\% of sample size). \\
\hline
\end{tabular}${ }^{+}$While the above procedures are listed as independent frequencies, \\
many overlap.
\end{tabular}


further operative details are listed in Table 2. Most patients underwent a total laparoscopic hysterectomy with bilateral salpingo-oopherectomy (98.7\%) and lymph node dissection (82.8\%). Other than cystotomy repair, no additional urinary or gastrointestinal procedures were planned or performed. Four cases $(2.5 \%)$ were converted to laparotomy. One case was due to large uterine size, another required radical debulking due to preliminary diagnosis of uterine sarcoma, another due to large vessel injury requiring immediate intervention, and the last due to poor access and visualization limiting completion of surgery laparoscopically. None of these cases included our patients sustaining a bladder or ureteral injury. The total urinary tract injury rate was $3.2 \%$, where 3 patients $(1.9 \%)$ sustained a bladder injury and 2 patients (1.3\%) sustained a ureter injury. The visual detection rate of the urinary tract injuries was $60 \%(3 / 5)$, while the cystoscopy detection rate was $0 \%$. More specifically, the visual detection rate of bladder injury was $100 \%(3 / 3)$.

Table 3 outlines the main characteristics of the 5 cases of urinary tract injury, along with the details of their diagnosis and management. In summary, Case 1 was a 61-year-old patient with a $14 \mathrm{~cm}$ size uterus who underwent surgical staging followed by a lower transverse incision for extraction of a large specimen that was complicated by a bladder dome cystotomy, which was immediately recognized and repaired in a standard 2-layer fashion. Cystoscopy confirmed the integrity of the bladder and ureters. Case 2 was an 89-year-old patient with a BMI of 32 who underwent surgical staging for grade-1 adenocarcinoma of the endometrium, which was complicated by dense peritoneal adhesions and retroperitoneal fibrosis. A cystotomy was sustained during bladder dissection. It was immediately recognized and repaired in a standard twolayer fashion. Cystoscopy confirmed the integrity of the bladder and ureters. Case 3 was a 57 -year-old patient with postmenopausal bleeding with endometrial biopsy showing complex atypical hyperplasia of the endometrium. She had a past surgical history of 2 cesarean sections. Her BMI was $34 \mathrm{~kg} / \mathrm{m}^{2}$, and her uterus was $14 \mathrm{~cm}$ in size. Adhesions were pervasive throughout the pelvis causing a frozen pelvis. A 1 $\mathrm{cm}$ cystotomy was noted during bladder dissection. This was repaired in two layers. A cystoscopy was performed to ensure the integrity of the ureters and the bladder. Case 4 was an 84-year-old patient with a BMI of 42 and a history of ruptured appendicitis who during surgical staging underwent extensive lysis of intraperitoneal adhesions as well as ureterolysis for dense retroperitoneal fibrosis. Cystoscopy at the conclusion confirmed bladder integrity and bilateral ureteral jets. At her 2-week postoperative check, she complained of leakage of clear fluid from the vagina. A CT urogram was performed noting left sided urinoma and left hydroureter. The patient proceeded for urologic evaluation and treatment, however she did not follow up with the surgeon. Finally, Case 5 was a 47-year-old patient with a BMI of $21 \mathrm{~kg} / \mathrm{m}^{2}$, who underwent an uneventful surgical staging procedure followed by cystoscopy to confirm the integrity of the bladder and ureters. She presented at her 2-week postoperative visit with complaints

Table 3: Urinary tract injury patient details, diagnoses, and management.

\begin{tabular}{|c|c|c|c|c|c|}
\hline & Case 1 & Case 2 & Case 3 & Case 4 & Case 5 \\
\hline Type of injury & Bladder & Bladder & Bladder & Ureteral & Ureteral \\
\hline Age & 61 & 89 & 57 & 84 & 47 \\
\hline BMI & 25 & 32 & 34 & 42 & 21 \\
\hline Prior surgery & + & - & - & + & - \\
\hline Preoperative diagnosis & $\begin{array}{l}\text { High grade } \\
\text { endometrial stromal } \\
\text { sarcoma }\end{array}$ & Grade 1 EAC & $\begin{array}{l}\text { Complex atypical } \\
\text { hyperplasia }\end{array}$ & Grade 2 EAC & Grade 1 EAC \\
\hline Procedure & TLHBSO & TLHBSO & RRHBSO & TLHBSO & TLHBSO \\
\hline LND & PPALND & PPALND & - & PPALND & PPALND \\
\hline Adhesiolysis & - & + & + & + & - \\
\hline Ureterolysis & + & - & + & + & + \\
\hline Other procedures & $\begin{array}{l}\text { Mini-laparotomy, } \\
\text { cystotomy repair }\end{array}$ & Cystotomy repair & $\begin{array}{l}\text { Proctosigmoidoscopy, } \\
\text { cystotomy repair }\end{array}$ & - & - \\
\hline Uterine size (g) & 158 & 70 & 225 & 82 & 222 \\
\hline Pathology & $\begin{array}{l}\text { Stage } 1 B \text {, Grade } 2 \\
\text { EAC }\end{array}$ & Stage $1 \mathrm{~B}$, Grade $1 \mathrm{EAC}$ & Stage $1 \mathrm{~A}$, Grade $1 \mathrm{EAC}$ & Stage 1B, Grade 2 EAC & $\begin{array}{l}\text { Stage } 1 \mathrm{~A} \text {, Grade } \\
1 \mathrm{EAC}\end{array}$ \\
\hline Blood loss (mL) & 150 & 250 & 250 & 50 & 150 \\
\hline Surgical time (min) & 250 & 256 & 215 & 209 & 204 \\
\hline Complications & $\begin{array}{l}\text { Cystotomy during } \\
\text { mini-laparotomy }\end{array}$ & $\begin{array}{l}\text { Cystotomy incurred } \\
\text { during dissection of } \\
\text { bladder from cervix }\end{array}$ & $\begin{array}{l}\text { Cystotomy incurred } \\
\text { during dissection of } \\
\text { bladder from cervix }\end{array}$ & $\begin{array}{l}\text { Left ureteral injury } \\
\text { detected } 2 \text { weeks after } \\
\text { surgery }\end{array}$ & $\begin{array}{l}\text { Left ureteral injury } \\
\text { detected } 2 \text { weeks } \\
\text { after surgery }\end{array}$ \\
\hline Management & $\begin{array}{l}\text { Repaired } \\
\text { intraoperatively- } \\
\text { confirmed bladder } \\
\text { integrity with } \\
\text { cystoscopy }\end{array}$ & $\begin{array}{l}\text { Repaired } \\
\text { intraoperatively- } \\
\text { confirmed bladder } \\
\text { integrity with } \\
\text { cystoscopy }\end{array}$ & $\begin{array}{l}\text { Repaired } \\
\text { intraoperatively- } \\
\text { confirmed bladder } \\
\text { integrity with cystoscopy }\end{array}$ & $\begin{array}{l}\text { Patient was treated } \\
\text { extramurally }\end{array}$ & $\begin{array}{l}\text { Patient had } \\
\text { outpatient } \\
\text { placement of left } \\
\text { ureteral stent }\end{array}$ \\
\hline
\end{tabular}

$\mathrm{EAC}=$ Endometrial adenocarcinoma; $\mathrm{TLH}=$ Total laparoscopic hysterectomy $; \mathrm{RRH}=$ Robotic radical hysterectomy; $\mathrm{BSO}=$ Bilateral salpingooophorectomy; PPALND = Pelvic and para-aortic lymph node dissection. 
Citation: El-neemany D, Pursell N, Curcio E, et al. (2019) Routine Cystoscopy during Robotic Hysterectomy for Endometrial Cancer. Reports Gynecol Surg 2(1):12-17

Table 4: Comparison of data by urinary tract (UT) injury status.

\begin{tabular}{|c|c|c|c|}
\hline & Patients w/o UT injury $(n=152)$ & Patients w/UT injury $(n=5)$ & P-Value \\
\hline Patient demographics: & \multicolumn{3}{|l|}{ Median [IQR] or Frequency (\%) } \\
\hline Age at surgery (years) & $66.1[57.1,74.3]$ & $61.9[57.2,84.5]$ & 0.92 \\
\hline Body mass index $\left(\mathrm{kg} / \mathrm{m}^{2}\right)$ & $33[28,40]$ & $32[25,34]$ & 0.40 \\
\hline History of CVD & $116(76.3)$ & $3(60.0)$ & 0.60 \\
\hline History of COPD & $40(26.3)$ & $2(40.0)$ & 0.61 \\
\hline Prior abdominal surgery & $74(48.7)$ & $2(40.0)$ & 1.0 \\
\hline EAC stage 1 & $109(71.7)$ & $5(100.0)$ & 0.33 \\
\hline Histopathology type 1 & $136(89.5)$ & $4(80.0)$ & 0.44 \\
\hline Primary procedure performed: & \multicolumn{3}{|l|}{ Frequency (\%) } \\
\hline Total laparoscopic hysterectomy & $147(96.7)$ & $4(80.0)$ & 0.18 \\
\hline Radical laparoscopic hysterectomy & $5(3.3)$ & $1(20.0)$ & \\
\hline Additional procedural stepst: & \multicolumn{3}{|l|}{ Frequency (\%) } \\
\hline Bilateral salpingectomy & $2(1.3)$ & $0(0.0)$ & 1.0 \\
\hline Bilateral salpingo-oophorectomy & $150(98.7)$ & $5(100.0)$ & 1.0 \\
\hline Omentectomy & $29(19.1)$ & $0(0.0)$ & 0.59 \\
\hline Lysis of adhesions & $87(57.2)$ & $3(60.0)$ & 1.0 \\
\hline Pelvic lymph node dissection & $124(81.6)^{*}$ & $4(80.0)$ & 1.0 \\
\hline Para-aortic lymph node dissection & $90(59.2)^{*}$ & $4(80.0)$ & 0.65 \\
\hline Procedural/outcome measures: & \multicolumn{3}{|l|}{ Median [IQR] } \\
\hline Uterine weight (g) & $140[96,188]^{*}$ & $158[82,222]$ & 0.98 \\
\hline Length of surgery (min) & $196[158,231.5]^{*}$ & $215[209,250]$ & 0.13 \\
\hline Estimated total blood loss (mL) & $100[100,150]^{*}$ & $150[150,250]$ & 0.09 \\
\hline Total length of hospital stay (days) & $1[1,1]$ & $1[1,1]$ & 1.0 \\
\hline
\end{tabular}

*Indicates missing data (less than $5 \%$ of sample size).

${ }^{+}$While the above procedures are listed as independent frequencies, many overlap.

of vaginal discharge. A CT urogram was performed and noted a left ureteral injury. She proceeded for urologic evaluation and placement of left stent. Her symptoms resolved without further complications. The stent was removed in 6 weeks.

A comparison of operative data between the patients with urinary tract injury versus those without is summarized in Table 4. There were no statistical differences found between the two groups in terms of median age $(p=0.92)$ and BMI ( $p$ $=0.40$ ), or existing comorbidities at the time of surgery (CVD: $p=0.60$; COPD: $p=0.61$; Prior abdominal surgery: $p=1.0$ ). Additionally, there were no statistical differences between the groups for stage $(p=0.33)$ and histopathology of cancer $(p=0.44)$, the type of surgery performed $(p=0.18)$, surgical time $(p=0.13)$, median uterine weight $(p=0.98)$, length of hospital stay $(p=1.0)$, or the frequency of any additional procedures performed such as pelvic lymph node dissection $(p=1.0)$, omentectomy $(p=0.59)$, or lysis of adhesions ( $p$ $=1.0$ ). Additionally, the total estimated blood loss was not found to be statistically different $(p=0.09)$ between the patients with a urinary tract injury (Median [Interquartile Range]: $150 \mathrm{ml}[150,250])$ compared to those without injury $(100 \mathrm{ml}[100,150])$.

\section{Comment}

The overall incidence rate of urinary tract injury during robotic staging for endometrial cancer in this study was $3.2 \%$, with 3 intraoperative bladder injuries and 2 postoperative ureter injuries occurring. Routine cystoscopy did not alter the detection rate of any of these injuries.
In a retrospective chart review of robotic surgery performed for malignant pathology at two different institutions, Nguyen, et al. reported no urinary tract injuries and hence did not find cystoscopy to be of benefit in detecting injuries in the routine cystoscopy group [9]. In the Gynecologic Oncology Group LAP2 study, one of the largest prospective studies of laparoscopic staging for endometrial cancer, the reported urinary tract injury rate was 2-3\% [15]. In another prospective study of 471 hysterectomy cases for benign disease reported by Vakili, et al. (10\% laparoscopic hysterectomy), the total urinary tract injury rate was $4.8 \%$ (1.7\% ureter injury, 3.6\% bladder injury) [16]. The authors argued that the incidence of urinary tract injury with hysterectomy might be underestimated since most of the literature comprises retrospective studies that may have been limited by the underreporting of cases that presented in the postoperative period. All but one patient in our study continue to be followed in our practice as part of cancer surveillance up to the date of writing of this manuscript.

In two studies by Sandberg and Nguyen, the incidence of urinary tract injury was $0 \%$ and up to $0.7 \%$, respectively $[8,9]$. Cystoscopy was not found to increase the detection rate of these injuries in either of these studies. The former recommended selective rather than universal cystoscopy, while the latter argued the importance of training physicians on the efficient use of cystoscopy in order to avoid potentially morbid and costly injuries as well as litigation [8,9]. In a large systematic review of the literature [3], Gilmour, et al. found a 5 -fold increase in injury rates when routine intraoperative cystoscopy was performed during laparoscopic hysterectomy 
with or without other gynecologic or other urogynecologic procedures. The authors reported that $89 \%$ of ureteric injuries and $95 \%$ of bladder injuries were detected when cystoscopy was routinely performed (vs. $7 \%$ and $43 \%$, respectively); However, the number of injuries detected with cystoscopy was unclear. In a follow up review [6], the authors maintained a 5 -fold increase in injury detection rates when cystoscopy was used intraoperatively, but found no evidence that cystoscopy provided any meaningful reduction in the number of postoperatively detected injuries. Additionally, in a recent systematic review of urinary tract injury in gynecologic laparoscopy for benign indications, bladder injuries were more commonly recognized intraoperatively and attributed to lysis of adhesions while ureter injuriesmuch like our observations- were more commonly recognized postoperatively and attributed to thermal injury [17].

Although we cannot definitively determine the cause of the delayed ureter injuries observed in our study, possible explanations include devascularization and thermal damage, especially since $100 \%$ of those injuries were detected postoperatively. It has been observed that thermal injuries often present between 2-14 days postoperatively, and that the diagnosis is often illusive due to the difficulty in differentiating thermal coagulative necrosis from longstanding inflammation and secondary infection [18]. Various mechanisms of laparoscopic energy source-related injuries have been described. Monopolar electrocautery-related injuries are associated with capacitive coupling, insulation failure, or direct coupling, which occurs when current from the active electrode passes to another instrument that is in contact with tissues away from the active electrode. Capacitive coupling can be induced in a conductor adjacent to the insulation surrounding the active electrode and can be generated along the insulation shaft of the active electrode with biologic fluid acting as a conductor, especially at high electrosurgical generator settings [18]. Monopolar and bipolar electrosurgical devices can also cause tissue damage through thermal spread. While bipolar electrosurgical spread is not well assessed, it is believed to range from $2 \mathrm{~mm}$ to 22 $\mathrm{mm}$. Interestingly, thermal effect as far as $1 \mathrm{~cm}$ away has been documented when the monopolar electrode tip was activated for greater than 10 seconds with a power setting of $40 \mathrm{~W}$ [18]. The power setting for both monopolar and bipolar electrocautery used in this study was arbitrarily set at $45 \mathrm{~W}$. While we cannot definitively prove causation between our injuries and electrosurgery unit settings, we certainly view our findings as an eye opener that are worth heeding and have since decreased our power settings especially when performing adhesiolysis and ureterolysis.

We do not believe that the lack of use of indigo carmine dye during cystoscopy has led to a decrease in our detection rate for a couple of reasons. First, cystoscopy was not terminated in any of the cases before the surgeon and his assistants confirmed satisfactory bilateral ureter jet streams. Second, the use of saline is certainly acceptable by gynecologists and urologists and has served as the control arm in published clinical trials aiming to find alternatives to indigo carmine $[19,20]$.

Risk factors associated with increased rates of urinary tract injury in previous studies included: The addition of prolapse procedures, the use of laparoscopic or robotic surgery, the presence of adhesions, and low volume surgeons $[8,16]$. We were unable to identify statistically significant risk factors that predict urinary tract injury in our study (Table 4), most likely due to the small number of injury cases. A descriptive synopsis of those cases is provided in the text (Table 3).

As with any retrospective study, there are limitations to our study. A small number of patients ( $5 \%$ or less) had missing data (noted in Table 1, Table 2 and Table 4). However, we note that all 5 cases with urinary tract injury had complete data available. Another limitation is that the results of this study reflect only a single gynecologic oncology surgeon's practice, hence generalization of the results should be made with caution. Additionally, given the low rate of urinary tract injury in the patient sample, few inferential statistical analyses could be done to correlate patient demographic, disease specific, and operative characteristics with the occurrence of urinary tract injury.

In conclusion, routine cystoscopy during robotic hysterectomy for endometrial cancer staging did not alter the detection rate of urinary tract injury in this study. Based on this study, the pertinence of cystoscopy in the case of bladder injury is restricted to evaluating the lower urinary tract after bladder repair. However, this finding cannot be extrapolated to hysterectomy for other indications such as pelvic reconstructive surgery. The potential net positive impact of performing cystoscopy vis a vis resident physician training should be considered.

\section{Disclosure}

The authors report no conflict of interest.

\section{Funding}

There was no funding sources used for this study.

\section{References}

1. AAGL advancing minimally invasive gynecology worldwide (2012) AAGL practice report: Practice guidelines for intraoperative cystoscopy in laparoscopic hysterectomy. J Minim Invasive Gynecol 19: 407-411.

2. Harkki-Siren $P$, Sjoberg J, Tiitinen A (1998) Urinary tract injuries after hysterectomy. Obstet Gynecol 92: 113-118.

3. Gilmour DT, Das S, Flowerdew G (2006) Rates of urinary tract injury from gynecologic surgery and the role of intraoperative cystoscopy. Obstet Gynecol 107: 1366-1372.

4. Aarts JW, Nieboer TE, Johnson N, et al. (2015) Surgical approach to hysterectomy for benign gynaecological disease. Cochrane Database Syst Rev 12.

5. Gilmour DT, Baskett TF (2005) Disability and litigation from urinary tract injuries at benign gynecologic surgery in Canada. Obstet Gynecol 105: 109-114.

6. Teeluckdharry B, Gilmour D, Flowerdew G (2015) Urinary tract injury at benign gynecologic surgery and the role of cystoscopy: A systematic review and meta-analysis. Obstet Gynecol 126: 1161-1169.

7. Chi AM, Curran DS, Morgan DM, et al. (2016) Universal 
cystoscopy after benign hysterectomy: Examining the effects of an institutional policy. Obstet Gynecol 127: 369-375.

8. Sandberg EM, Cohen SL, Hurwitz S, et al. (2012) Utility of cystoscopy during hysterectomy. Obstet Gynecol 120: 13631370.

9. Nguyen ML, Stevens E, LaFargue CJ, et al. (2014) Routine cystoscopy after robotic gynecologic oncology surgery. JSLS 18.

10. Tan SJ, Lin CK, Fu PT, et al. (2012) Robotic surgery in complicated gynecologic diseases: Experience of tri-service general hospital in Taiwan. Taiwan J Obstet Gynecol 51: 18-25.

11. Lambaudie E, Houvenaeghel G, Walz J, et al. (2008) Robotassisted laparoscopy in gynecologic oncology. Surg Endosc 22: 2743-2747.

12. Zanagnolo V, Garbi A, Achilarre MT, et al. (2017) Robot-assisted surgery in gynecologic cancers. J Minim Invasive Gynecol 24: 379-396.

13. Barrie A, Freeman AH, Lyon L, et al. (2016) Classification of postoperative complications in robotic-assisted compared with laparoscopic hysterectomy for endometrial cancer. J Minim Invasive Gynecol 23: 1181-1188.

14. Gehrig PA, Cantrell LA, Shafer A, et al. (2008) What is the optimal minimally invasive surgical procedure for endometrial cancer staging in the obese and morbidly obese woman? Gynecol Oncol 111: 41-45.

15. Walker JL, Piedmonte MR, Spirtos NM, et al. (2009) Laparoscopy compared with laparotomy for comprehensive surgical staging of uterine cancer: Gynecologic oncology group study LAP2. J Clin Oncol 27: 5331-5336.

16. Vakili B, Chesson RR, Kyle BL, et al. (2005) The incidence of urinary tract injury during hysterectomy: $A$ prospective analysis based on universal cystoscopy. Am J Obstet Gynecol 192: 15991604.

17. Wong JM, Bortoletto $P$, Tolentino J, et al. (2018) Urinary tract injury in gynecologic laparoscopy for benign indication: A systematic review. Obstet Gynecol 131: 100-108.

18. Law KS, Abbott JA, Lyons SD (2014) Energy sources for gynecologic laparoscopic surgery: A review of the literature. Obstet Gynecol Surv 69: 763-776.

19. Grimes CL, Patankar S, Ryntz T, et al. (2017) Evaluating ureteral patency in the post-indigo carmine era: A randomized controlled trial. Am J Obstet Gynecol 217: 601.

20. Espaillat-Rijo L, Siff L, Alas A, et al. (2016) Intraoperative cystoscopic evaluation of ureteral patency: A randomized controlled trial. Obstet Gynecol 128: 1378-1383. 